\title{
Slough: what does it mean and how can it be managed
}

Angel D

Keywords Slough, necrotic tissue, debridement, desloughing, wound bed preparation

For referencing Angel D. Slough: what does it mean and how can it be managed. Wound Practice and Research 2019; 27(4):164-167.

DOI https://doi.org/10.33235/wpr.27.4.164-167

\section{ABSTRACT}

The purpose of this paper is to provide an overview of the formation of slough on the wound bed. The impact slough has on wound healing will also be explored. There are several wound cleansing products which can be used for the safe removal of slough, and several different methods of debridement - including autolytic, conservative sharp, surgical, ultrasonic, hydrosurgical and mechanical - as well as several therapies which can be used, including osmotic, biological, enzymatic dressings and monofilament fibre pads. These various methods will be described.

\section{BACKGROUND}

Slough is a common feature of chronic wounds, although the number of wounds that contain slough has not yet been reported in the literature ${ }^{1}$. In certain circumstances, such as fungating malignant wounds, wound management is usually palliative with comfort measures. Conversely, if the goal of care is wound healing, the removal of slough is a vital component of wound bed preparation?

Fundamentally, acute wounds go through an orderly sequence of wound healing, which includes haemostasis, inflammation, repair and remodelling of scar tissue. Each phase overlaps and directs the next phase of wound healing ${ }^{2,3}$. There is a controlled release of growth factors, cytokines and proteases which control cell migration, differentiation and proliferation'. However, certain wounds do not progress through the predicted wound healing trajectory, becoming chronic wounds. They have a tendency to remain in the inflammatory phase of wound healing ${ }^{3}$. This could be as a

\section{Donna Angel}

RN, NP, BN, PGDip (Clin Spec), MSc (Nur)

Royal Perth Hospital

Wellington Street, Perth, WA

Email Donna.angel@health.wa.gov.au result of infection, or repeated ischaemia and reperfusion injury which in turn leads to prolonged inflammation ${ }^{4}$. This results in high levels of matrix metalloproteases (MMPs) reducing the number of growth factors produced, leading to cellular senescence. The wound exudate becomes toxic to the extracellular matrix. This prolonged inflammation increases phagocytosis and apoptosis, which potentially increases the presence of slough on the wound bed ${ }^{4-6}$. Typical examples of chronic wounds are pressure injuries, venous leg ulcers and diabetic foot ulcers ${ }^{1}$.

Slough is essentially the by-product of the inflammatory phase of wound healing comprising of fibrin, leucocytes, dead and living cells, microorganisms and proteinaceous material ${ }^{1}$. The appearance of slough is typically a pale yellow, viscous fibrinous tissue and can range from yellow to tan, usually, but not always, covering the entire wound bed. It can appear on parts of the wound bed and tends to be either loosely adhered to the surface of the wound or firmly attached ${ }^{1,7-9}$. Slough attracts bacteria to the wound surface, resulting in low levels of inflammation. White blood cells and plasma infiltrate the wound bed causing peri-wound oedema, increased levels of exudate and an acceleration in cellular activity ${ }^{7}$. Biofilms are also present in the majority of chronic wounds, contributing to delayed wound healing ${ }^{10,11}$. Percival and Suleman postulate that there is a direct link between slough and the formation of biofilms on the wound bed'.

\section{SLOUGH AS A BARRIER TO WOUND HEALING}

The appropriate and safe removal of slough is a vital component of wound healing ${ }^{12}$. Slough on a wound bed not only makes it difficult for clinicians to assess the wound bed accurately, it also contributes to delayed wound healing. The presence of slough also ${ }^{1,13}$ :

- Prolongs the inflammatory response, resulting in high levels of protease and pro-inflammatory cytokine production.

- Provides a focus for infection.

- Mimics/hides infection. 
- Attracts bacteria to the wound.

- Increases odour and exudate.

- Prevents the wound from progressing through the wound healing process.

\section{DESLOUGHING VERSUS DEBRIDEMENT}

There is controversy in the literature regarding the safe removal of devitalised tissue by either desloughing or debridement strategies. It has been suggested that there is no difference with either method ${ }^{14}$. Necrotic tissue is associated with cell death resulting from injury, infection and/ or an underlying disease process. Classically, necrotic tissue is black in colour; however, it can also present as brown or grey, and is typically dry although this can change depending on hydration. Necrotic tissue will either be firmly attached or separating from the wound margins ${ }^{9}$. Slough is considered to be part of the inflammatory process consisting of fibrin, white blood cells, bacteria and debris, along with dead tissue and other proteinaceous material. It is the cellular debris resulting from the process of inflammation ${ }^{7}$. Unlike necrotic tissue, the goal of care with sloughy tissue is usually to remove it. Necrotic tissue should not be removed in the presence of untreated arterial disease, gangrene, stable eschar on heels (unless there is adequate tissue perfusion), fungating or ulcerating tumours and wounds with an underlying inflammatory process such as pyoderma gangrenosum ${ }^{15}$. As necrotic tissue is an entity in itself, this paper will focus solely on the impact of slough on the wound bed and approaches for removal.

Natural desloughing is the endogenous action of enzymes produced from white blood cells; essentially they soften and liquefy devitalised tissue - this is know as autolysis. Assisted desloughing occurs when the body's own natural autolytic processes are unable to cope with the quantity of tissue damage. A number of different methods are used to facilitate autolysis ${ }^{7}$. Desloughing is considered a lower risk alternative to debridement; this method includes the use of wound cleansing agents and wound care products, whereas debridement is usually reserved for necrotic tissue. The method chosen is dependent on a comprehensive wound assessment including clinical need, underlying comorbidities such as clotting disorders, and the underlying pathology of the wound.

However, there is disagreement over the difference between desloughing and debridement as both terms are used interchangeably in the literature causing a degree of confusion ${ }^{1}$. At the European Wound Management Association (EWMA) conference in London 2015, a debate was held to determine if there was a difference. The debate was lead by three leading tissue viability nurses and sponsored by Urgo Medical ${ }^{14}$. The key points that came out of the debate were that:

- Creating a new category runs the risk of confusing nurses.

- Desloughing is part of debridement.
- Clinical education is required - this must relate to patient assessment and be the most appropriate technique to use.

- Clinicians must be able to assess and differentiate between slough and necrotic tissue.

\section{CONSIDERATIONS BEFORE DEBRIDEMENT AND CLINICAL CHALLENGES}

Slough is present in the large majority of chronic wounds. Slough recurrence is common post-debridement/ desloughing, therefore ongoing desloughing needs to be maintained ${ }^{1}$. There is no single method able to remove all devitalised tissue. Instead, evidence suggests that a combination of methods is required ${ }^{7}$.

Prior to debridement, the procedure must be fully explained to the patient and consent gained. The method chosen will depend on a number of factors including but not limited to: the underlying pathology of wound, comorbidities, the potential risk for bleeding, the extent of the sloughy tissue small versus a large area, the level of pain the patient is experiencing, the resources available, the level of skill of the healthcare practitioner, and whether the procedure will be performed in a hospital versus community setting ${ }^{15,16}$. Having established that removal of slough is vital for wound healing, the clinician must determine which is the best approach to take.

There are also several wound cleansing products available on the market, and so it is important to consider which is the most suitable. Some contain surfactants and antimicrobial agents, while others are super-oxidised, containing either low levels of hypochlorous acid or sodium hypochlorite. Wound cleansing agents help to loosen devitalised tissue and either prevent biofilm attachment or disrupt biofilms ${ }^{17}$. Wound cleansing is a vital component of wound bed preparation.

METHODS OF DESLOUGHING / DEBRIDEMENT

Autolytic debridement: Selective debridement is the release of the body's own proteolytic enzymes and phagocytes which liquefy and soften the devitalised tissue ${ }^{9}$. The nonviable tissue is selectively liquefied, separated and digested by endogenous enzymes ${ }^{9}$. Dressings support autolytic debridement by providing a moist wound environment ${ }^{1,16}$. The choice of dressing is reliant on a comprehensive wound assessment and the goal of care. The clinician must have an understanding of the properties of the dressing that is chosen, indications for use and contraindications, and at all times follow the manufacturers' instructions. Be aware that some patients are allergic or sensitive to certain dressing products and that certain dressings can cause pain. With this in mind, it is important to include the patient where possible on the selection of a dressing.

As a guide, if the wound has dry adherent slough on the wound bed, select a dressing that will donate moisture. If the wound is sloughy and exudating, then select a dressing that 
is absorbent, being mindful that it needs to absorb enough exudate to minimise the risk of peri-wound maceration. Alternatively, dressing change frequency may need to be increased ${ }^{15,16}$. In wounds that are demonstrating signs of local infection ${ }^{17}$ such as erythema, local warmth, swelling, purulent discharge, delayed wound healing, new or increase in pain and/or increase in malodour ${ }^{17}$, it is prudent to use an antimicrobial dressing.

Autolytic debridement is considered safe, selective and relatively painless ${ }^{16,18}$; however, this method can take time, with several dressing changes required. Autolytic debridement is not indicated for patients with ischaemic limbs or digits where the goal of care is to keep the wound $\mathrm{dry}^{15}$, nor for clinically infected or deep cavity wounds ${ }^{15,18}$. If the wound is clinically infected, surgical debridement should be considered ${ }^{16,18}$.

Conservative sharp debridement: This is the selective removal of non-viable tissue using sharp instruments, for example a scalpel, scissors or a curette. This technique can be performed at the bedside, outpatient setting or in the community. Care must be taken to avoid damage to the underlying tissue. The practitioner must also be aware of prosthetic devices beneath the devitalised tissue ${ }^{12}$. This method has similar risks to surgical debridement; however, the degree of risk is less ${ }^{15}$. Perform this procedure only if it is within your scope of practice, you have the proficiency to perform the procedure, and you know when to stop ${ }^{18}$. Ensure an aseptic technique is maintained during the procedure at all times.

Surgical debridement: This is a non-selective method of debridement due to the need to have clear margins to healthy tissue $^{19}$, and is performed in an operating theatre using regional or general anaesthetic by a surgeon, usually from the specialty of plastics, vascular, trauma or a general surgeon ${ }^{16}$. It is indicated for wounds with extensive tissue necrosis or infection. If the facility does not have the necessary personnel nor equipment urgent, referral to a tertiary hospital may be required for patients with sepsis or necrotising fasciitis ${ }^{18}$.

There are additional risks associated with anaesthesia. For example, caution should be exercised in patients with diabetes, arterial disease and clotting disorders ${ }^{15}$. There is also an increased risk of bleeding, pain and damage to underlying structures. However, this method is a quick and efficient method of debridement ${ }^{15}$.

Ultrasonic debridement: This is offered via a low frequency ultrasound $(25-30 \mathrm{KHz})$ delivered via a saline or wound cleansing solution. There are two modes - contact and non-contact. Contact mode debrides the wound without damaging healthy tissue, has a bactericidal effect, disrupts biofilms, and stimulates wound healing. Non-contact mode has a bactericidal effect only ${ }^{20,21}$. This type of debridement can be used on a number of different wound and tissue types, including diabetic foot wounds, venous leg ulcers, wounds deemed not suitable for surgery, and where tendon, connective tissue and bone are exposed ${ }^{16,22}$. This procedure requires trained personnel. Protective clothing also needs be worn due to the risks associated with aerosolisation of blood products and microorganisms ${ }^{23}$. The initial set up of the machine and equipment can be costly; the hand pieces are not disposable and need to be sterilised ${ }^{20}$.

Hydrosurgical debridement: This combines physical and surgical debridement. Using a high pressure jet of saline creates a Venturi effect - the movement of fluid through a constricted opening, resulting in a decrease in pressure and a suction effect - which enables the removal of necrotic tissue via a disposable handpiece ${ }^{1}$. However, unlike ultrasonic debridement, it is not selective and will therefore remove healthy and devitalised tissue. As with ultrasonic debridement, the equipment can be costly, requires trained personnel, and there are also risks associated with aerosolisation, therefore protective clothing must be worn ${ }^{19}$.

Mechanical debridement: The traditional method of wet to dry dressings is no longer advocated as a method of removing slough. This involves using wet to dry gauze on a wound. The top layer adheres to the wound bed; however, when removed, both healthy and unhealthy tissue is removed. It can also be painful and traumatic for the patient ${ }^{19,23}$. This method can cause prolonged inflammation, damage healthy tissue, and potentially increase the risk of infection, resulting in delayed wound healing ${ }^{15}$.

Osmotic therapy (honey): The type of honey used is specifically manufactured for use in wound care. It is derived from selected Leptospermum or Eucalyptus marginata and Santalum species of plants and registered with the Therapeutic Goods Administration for use in open wounds ${ }^{24}$. Honey draws fluid from the surrounding tissue to accelerate autolytic debridement. The wound $\mathrm{pH}$ is reduced to 3.0-4.5. This creates an acidic environment which is hostile to bacteria, particularly Pseudomonas aeruginosa or Staphylococcus aureus ${ }^{16,24}$. A by-product is the release of hydrogen peroxide or methylglyoxal; this also supports autolytic debridement ${ }^{21}$.

Biological (laval) therapy: Live sterile maggots derived from Lucilia sericata species are introduced into the wound bed either loose or in net dressings. The maggots eat the devitalised tissue, secreting an antibacterial compound. This reduces bio burden and inflammation within the wound ${ }^{16,25}$. This method is considered selective, as the maggots do not eat healthy tissue. For optimal results, the wound must be kept moist by moistening the outer dressing at least daily. On completion of the treatment, the larvae need to be double bagged and incinerated ${ }^{19}$.

Enzymatic therapy: These are dressings derived from proteolytic enzymes which have been extracted from bovine plasma or pancreas, fruit and plants such as papin or bromelaine from pineapple, or bacteria collagenase derived from Clostridium histolyticum sp. They are recommended for 
hard dry eschar which may need to be scored with a scalpel to allow the enzyme to penetrate ${ }^{16}$. Currently these particular products are not available on the Australian market ${ }^{15}$. An enzyme alginogel is available, an alginate gel containing naturally occurring enzymes glucose oxidase (also found in honey) and lactoperoxidase (found in mammals' tears, mucous and milk), thereby producing an antibacterial effect $^{9,24}$.

Monofilament fibre pad therapy: In these dressings, the wound contact side is fleecy, comprising of monofilament polyester fibres (18 million per $10 \times 10 \mathrm{~cm})$. The pad needs to be pre-moistened with a wound cleansing solution, then gently wiped over the surface of the wound in a circular motion. The devitalised tissue is retained within the hoops of the dressing, thereby removing the debris ${ }^{19}$. It is useful for sloughy wounds and hyperkeratosis ${ }^{16}$. The pads are single patient use; they are not reusable.

\section{CONCLUSION}

It is vital that clinicians are able to differentiate between necrotic tissue and slough because the management of both tissue types can be different. Unlike sloughy tissue, where the goal of care is typically to remove it, there are instances where necrotic tissue should not be removed, or removed with caution.

Slough is considered the by-product of the inflammatory phase of wound healing. An essential component of wound bed preparation is the removal of slough from a wound bed. Slough not only contributes to delayed wound healing, it also prevents an accurate wound assessment and can also harbour biofilms. Not one method is suitable for all wounds. As a general rule, different removal methods are required. The decision as to which is the best approach for the removal of slough relies on a thorough assessment of the patient and the wound. Slough has a tendency to recur, therefore ongoing strategies need to be in place for ongoing removal. Removal of slough is a key step in promoting wound healing. The clinician needs to have a comprehensive understanding of what slough is, the impact on wound healing, and the various approaches that can be taken.

\section{CONFLICT OF INTEREST}

There are no conflicts of interest by the author.

\section{FUNDING}

The authors received no funding for this study.

\section{REFERENCES}

1. Percival S, Suleman N. Slough and biofilm: removal of barriers to wound healing by desloughing. J Wound Care 2015;24(11):498, 500-3, 6-10.

2. Schultz G, Sibbald G, Flanga V, Ayello E, Dowsett C, Harding K, et al. Wound bed preparation: a systematic approach to wound management. Wound Repair Regen 2003;11(Suppl 1):1-28.

3. Schultz G, Dowsett C. Wound bed preparation revisited. Wounds International 2012;3(1):27-9.
4. Mustoe T. Understanding chronic wounds: a unifying hypothesis on their pathogenesis and implications for therapy. Am J Surg 2004;187(5A):655-705.

5. Telegenhoff $D$, Shroot B. Cellular senescence mechanisms in chronic wound healing. Cell Death Different 2005;12:695-8.

6. Attinger C, Janis J, Steinberg J, Schwartz J, Al-Attar A, Couch K. Clinical approach to wounds: debridement and wound bed preparation including the use of dressings and wound healing adjuvants. Plastic Reconstruct Surg 2006;117(Suppl 7):72S-109S.

7. Milne J. Wound bed preparation: the importance of rapid and effective desloughing to promote healing. Br J Nurs 2015;24(Sup 20):S52-S8.

8. Gray D, White R, Cooper P. Applied wound management and using the wound healing continuum in practice. Wound Essentials 2010;5:131-9.

9. White W, Asimus M. Assessment and management of nonviable tissue. In: Swanson T, Asimus M, McGuiness B, editors. Wound management for the advanced practitioner. Melbourne: IP Communications; 2014. p. 170-203.

10. James G, Swogger E, Wolcott R, deLancey Pulcinic E, Secor $P$, Sestrich J, et al. Biofilms in chronic wounds. Wound Repair Regen 2008;16(1):37-44.

11. Bjarnsholt B, Kirketerp-Møller K, Jensen $\varnothing$, Madsen K, Phipps $\mathrm{R}$, Krogfelt $\mathrm{K}$, et al. When chronic wounds will not heal: a novel hypothesis. Wound Repair Regen 2008;16(1):2-10.

12. Ousey K, Mclntosh C. Understanding wound bed preparation and wound debridement. $\mathrm{Br} \mathrm{J}$ Community Nurs 2010;15(Suppl1):S22-8.

13. Leaper D, Schultz G, Carville K, Fletcher J, Swanson T, Drake R. Extending the TIME concept: what have we learned in the past 10 years? Int Wound J 2012;9(Suppl, 2):1-19.

14. Cowan T. Is there a difference between debridement and desloughing? Br J Nurs 2015;24(15):S18, S20.

15. Heath K, Angel D, Campbell C, Jackson M, Kourtis S, Legg A, et al. Ulcer and wound management. Melbourne: Therapeutic Guidelines Limited; 2019.

16. Strohal R, Apelgvist J, et al. EWMA document: debridement. J Wound Care 2013;22(Suppl 1):S1-S52.

17. International Wound Infection Institute (IWII). Wound infection in clinical practice. London: Wounds International. 2016.

18. Ayello E, Cuddigan J. Debridement: controlling the necrotic/ cellular burden. Adv Skin Wound Care 2004;17(2):66-75.

19. Leak K. Ten top tips for debridement. Wounds International 2012;3(1):21-3.

20. Butcher G, Pinnuck L. Wound bed preparation: ultrasonic debridement. Br J Nurs 2013;22(6):S36-S42.

21. Martínez J, Álvaro-Afonso F. Debridement and the diabetic foot. Foot Int 2019(Feb):2-10.

22. Gibbons G, Orgill D, TE S, Novoung A, O'Connell J, WW L, et al. A prospective randomized controlled trial comparing the effects of noncontact, low frequency ultrasound to standard care in healing venous leg ulcers. Ostomy Wound Manage 2015;61(1):16-29.

23. Gary D, Acton C, Chadwick P, Fumarola S, leaper D, Morris C, et al. Consensus guidance for the use of debridement techniques in the UK. Wounds UK 2011;7(1):77-84.

24. Carville K. Wound care manual. 7th ed. Perth, WA: Silver Chain Nursing Association; 2017.

25. Sig A, Koro O, Araz E. Maggot debridement therapy: utility in chronic wounds and a perspective beyond. Wound Pract \& Res 2017;26(3):140-5. 